\title{
Combined Location Routing and Inventory Problem of E-Commerce Distribution System with Fuzzy Random Demand
}

\author{
Dehui Chen ${ }^{1,3}$, Dongyan Chen ${ }^{2, *}$ Guanglu Sun ${ }^{4}$ and Guangming Liu ${ }^{4}$ \\ ${ }^{1}$ School of Management, Harbin University of Science and Technology, \\ Haerbin, China, 150080 \\ ${ }^{2}$ School of Applied Science, Harbin University of Science and Technology, \\ Haerbin, China, 150080 \\ ${ }^{3}$ School of Computer and Information Engineering, Harbin University of Commerce, \\ Haerbin, China, 150028 \\ ${ }^{4}$ School of Computer Science and Technology, Harbin University of Science and \\ Technology, Haerbin, China, 150080 \\ lcdh200811@163.com • \\ *Corresponding author: dychen_2004@yahoo.com.cm \\ Abstract
}

A fuzzy random mixed 0-1 integer programming model of Combined Location Routing and Inventory Problem (CLRIP) has been developed in B2C E Commerce distribution system. Demands of customers and distribution centers have been assumed to be fuzzy random variables. The expected value of fuzzy random axtable and the possibilistic mean value method have been used to transform the fuzzy random variables into determined ones. Therefore, this fuzzy random model has been converted into deterministic. A two-stage heuristic algorithm based on Tabu Search (TS) has been designed for solving determined model. A numerical example has demonstrated the effectiveness of the model and algorithm.

Keywords: distribution, location, routing, inventory, fuzzy random, Tabu Search

\section{Introduction}

Enterprises to build distribution system have three levels of decision-making: strategic, tactical and operational level. In the system design phase, location and other strategic layer decisions play an important role. Once the system frame is determined, the focus can be shifted to tactical and operational level decisions, such as inventory control and Vehicle Route Problens (VRP). In previous literature, most of the different levels of decision-making are considered separately. However, the decisions for solving these three levels are interrelated to one another. Therefore, we should fully consider combined location routing and in ventory problem (CLRIP) [1] in order to achieve the total distribution system cost savings.

In recent years, with the continuous occurrence of new algorithm, the research on logistics system optimization has focused on the integrated optimization with Location Inventory Problem (LIP), Location Routing Problem (LRP) and Inventory Routing Problem (IRP), which are all the two levels of decision. At present, there are few studies on this complex CLRIP. S.C. Liu and S.B. Lee (2003) made this study on CLRIP earliest. They constructed multi-depot LRP model taking inventory control decisions into consideration, and designed a two-phase heuristic method to solve this problem [1]. After that, a few scholars have been developing related research. As far as our best known, mostly works researched uncertainty 
demand in this field. Among them, the most studied CLRIP with random demand [2-6], only the few discussed CLRIP with fuzzy demand [7].

But in practice, customer demands are random and fuzzy. Currently, further research about fuzzy random variable (FRV) has been made in Supply Chain Management. Many scholars have studied FRV problem in logistics. Some scholars made a research on inventory optimization with FRV [8-13], and others studied routing optimization [14-15]. In general, all above researched single-level decision-making with FRV. There is little research on integrated optimization with FRV which combined two levels of decision. Especially, there is nearly no researcher who studied the CLRIP with FRV. Thus, this paper establishes a CLRIP model with fuzzy random demand (FRD) of B2C E-Commerce distribution system, and inventory decision of Distribution Center (DC) is agent to the supplier by introducing Vendor Management Inventory (VMI) to make enterprises meet customers' needs much faster and better.

\section{FRV and its Expectation}

A FRV is a measurable function from a probability space to a collection of fuzzy variables, and essentially a random variable taking fuzzy values [0. Since Kwakernaak (1978) first proposed the concept of FRV, and then different definitions of FRV were given on the basis of its different testability, therein, the definition given by B.D. Liu has been used.

Definition 2.1.[16] Let $\xi$ be a function from a probability space $\left(\Omega, \mathrm{A}, P_{r}\right)$ to a collection of fuzzy variables, if for any R on the Borel set B, $\operatorname{Pos}\{\xi(\omega) \in B\}$ is a measurable function of $\omega$, then $\xi$ is a FRV.

Combining the expectations definition of discrete FRV [10] and the expectations definition of fuzzy variables given by Liu 1171. ve present an expectations definition of discrete FRV in this paper.

\section{Definition 2.2. Let demand}
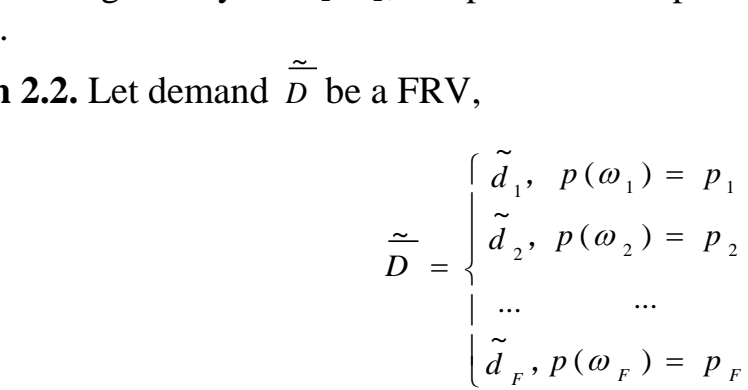

then $E(\tilde{\bar{D}})=\sum^{F} \tilde{E}\left(\tilde{d}_{f}\right) p_{f}$ is called the fuzzy random expectations of $\tilde{\bar{D}}$, where, $E\left(\tilde{d}_{f}\right)$ is the fuzzy expectations of fuzzy variable $\tilde{d}_{f}$, and

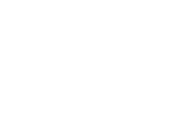

$$
E\left(\tilde{d}_{f}\right)=\int_{0}^{+\infty} \operatorname{Cr}\left\{d_{f} \geq r\right\} d r-\int_{-\infty}^{0} \operatorname{Cr}\left\{d_{f} \leq r\right\} d r
$$

provided that at least one of the two integrals is finite. Especially, if $\tilde{d}_{f}$ is a standard triangular fuzzy number $\left(\underline{d}_{f}, d_{f}, \bar{d}_{f}\right)$, then according to the work of [17], its fuzzy expectation is given by $E\left(\tilde{d}_{f}\right)=\frac{1}{4}\left(\underline{d}_{f}+2 d_{f}+\bar{d}_{f}\right)$. 


\section{Description and Modeling about CLRIP with FRV}

In VMI environment, there is often a longer order and production cycle from suppliers to enterprises. Thus, market conditions may change. For example, demand may increase, similar or decrease compared to the forecast demand. The demand for a variety of market circumstances can be given by the managers' experiences, and the probability distribution can be obtained by the historical data Statistics. This demand description only by random variable or fuzzy variable is not accurate. Instead, to describe it by FRV is more appropriate. For example, in the next order time, commodity demand may be increasing, similar or decreasing. If it increases, it can reach 10,000 units or so. If it is similar to forecast demand, it can reach 6,000 units or so. If it decreases, it can reach 3,000 units or so. At the same time, the probability of increasing is 0.5 , similar to forecast demand 0.3 and decreasing 0.2 . Now, we can only use the FRV to characterize demand, i.e. demand is given by [11].

$$
\tilde{\bar{D}}=\left\{\begin{array}{l}
\tilde{d}_{1}, p\left(\omega_{1}\right)=0.5 \\
\tilde{d}_{2}, p\left(\omega_{2}\right)=0.3 \\
\tilde{d}_{3}, p\left(\omega_{3}\right)=0.2
\end{array}\right\}
$$

Where $\tilde{\bar{D}}$ denotes FRD, $\omega_{1}, \omega_{2}$ and $\omega_{3}$ respectively denoted demand increasing, similar and decreasing compared to forecast demand, $\tilde{d}_{1}, \tilde{d}_{2}$ and $\tilde{d}_{2}$ denoted fuzzy demand, in general, fuzzy demand using standầd triangular fuzzy numbers, for example, $\tilde{d}_{f}=\left(\underline{d}_{f}, d_{f}, \bar{d}_{f}\right)(f=1,2,3)$ to represent the case, of the $\mathrm{f}$ kind of market demands of customers, the membership function of $d_{f}$ is as follows:

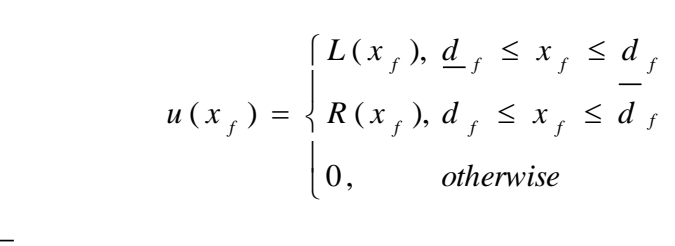

Where, $\underline{d}, d_{f} d_{f}$ are rea1numbers. $L\left(x_{f}\right), R\left(x_{f}\right)$ are the left and right shape functions, respectively. Here, $L\left(x_{f}\right)=\left(x_{f}-\underline{d}_{f}\right) /\left(d_{f}-\underline{d}_{f}\right) \quad, \quad R\left(x_{f}\right)=\left(\bar{d}_{f}-x_{f}\right) /\left(\bar{d}_{f}-d_{f}\right)$. Triangular fuzzy numbers $\tilde{d}_{f}$ may also be denoted by its $\alpha$-cut $d_{f}=\left[d_{f \alpha}^{-}, d_{f \alpha}^{+}\right], \alpha \in[0,1]$. Where, $d_{f \alpha}^{-}=\underline{d}_{f}+\alpha\left(d_{f}-\underline{d}_{f}\right), d_{f \alpha}^{+}=\bar{d}_{f}-\alpha\left(\bar{d}_{f}-d_{f}\right)$.

\subsection{Problem Description}

CLRIP model is to optimize the B2C E-Commerce distribution system in VMI environment, which consists of a single supplier, multiple DCs and a number of potential customers. Its network structure is shown in Figure 1. In this network, the first level is from supplier to DCs, the second level is from DCs to potential customers. Assuming that the location and number of candidate DCs and customers are determined, customer's demand is FRV. DC maintains a certain inventory, and its inventory decision given by supplier. As the supplier usually has a certain production cycle and uses the regular order model, this paper uses the periodic review inventory strategy, and touring distribution methods. We need to 
determine the number and location of DCs, vehicle routing for its allocated customers, optimal order time and the target inventory level of each open DC.

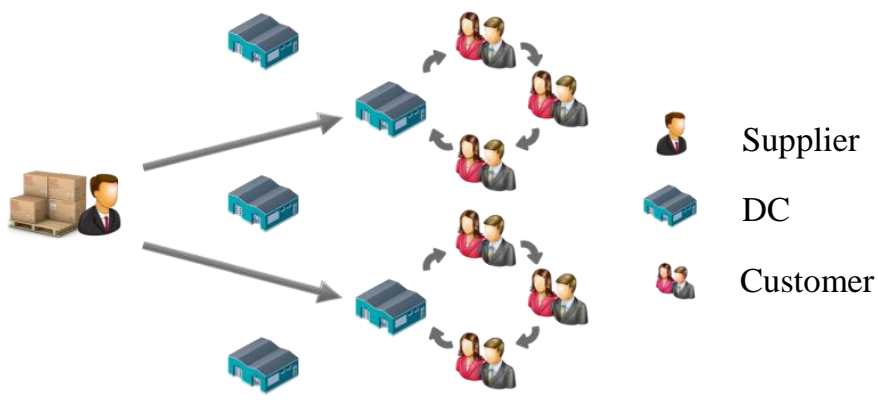

Figure 1. B2C E-Commerce Distribution System Network Structure

\subsection{Assumptions}

Main assumptions are as follows: the distribution goo OADC is single commodity; select the optimal number and location from the candidate DCs, and the annual opening and operating cost of each DC is known. Each customer can be served by only one vehicle. Each route has only one vehicle to service customers, and vehicles capacities are the same. The total demands of customers on each route car t exeeed the capaeity of the vehicle. Each route starts from a DC and ends at the same DC. Customers demand is independent and uncertain, which is considered as FRV. Each DC follows ( $T r$ seriodic review inventory policy.

\subsection{Parameters and Notations}

$\begin{array}{ll}I & \text { set of customers, } I=\{i \mid i=1,2, \cdots, n \\ J & \text { set of candidate DCS }\}=\{j \mid j=12, \cdots, m\} \\ K & \text { set of vehicles, } K=\{k \mid k=1,2 \cdots, l\} \\ F_{j} & \text { annual opening and operating cost of DC } j\end{array}$

$C_{l} \quad$ unit commodity transportation cost from supplier to each DC

$C_{2} \quad$ unit distance distribution cost from every DC to each customer

$C_{3} \quad$ cost per order placed to the supplier by each DC

$L \quad$ lead time

$T \quad$ order time

$d_{g h} \quad$ distance from node $g$ to node $h$

$C_{q} \quad$ delivery capacity of a vehicle

$\tilde{\bar{D}}_{j} \quad$ anual fuzzy random demand of customers served by DC $j$

$\tilde{\bar{D}}_{L}$ fuzzy random demand of customers served by DC $j$ at lead time, $\tilde{\bar{D}}_{L, j}=\tilde{\bar{D}}_{j} \times L$

$\tilde{\bar{D}}_{T, j}$ fuzzy random demand of customers served by DC $j$ at one period, $\tilde{\bar{D}}_{T, j}=\tilde{\bar{D}}_{j} \times T$

$\tilde{\bar{D}}_{L+T, j}$ fuzzy random demand of customers served by DC $j$ at lead time plus one period, $\tilde{\bar{D}}_{L+T, j}=\tilde{\bar{D}}_{j} \times(L+T)$

$\tilde{\bar{d}}_{i} \quad$ yearly fuzzy random demand of customer $i$

$r_{j} \quad$ target inventory level of $\mathrm{DC} j$ 
$C_{h} \quad$ inventory holding cost per unit commodity per year

$C_{s} \quad$ backorder cost per unit commodity per time

\subsection{Decision Variables}

$$
\begin{aligned}
& x_{g h k}= \begin{cases}1, & \text { if vehicle } k \text { is from node } g \text { to node } h \\
0, & \text { otherwise }\end{cases} \\
& y_{j}=\left\{\begin{array}{ll}
1, & \text { if } D C \quad j \text { is opened } \\
0, & \text { otherwise }
\end{array} \quad j=1,2, \cdots, m\right. \\
& z_{i j}=\left\{\begin{array}{ll}
1, & \text { if customer } i \text { is assigned to } D C j \\
0, & \text { otherwise }
\end{array} \quad i=1,2, \cdots, n, j=1,2, \cdots, m\right.
\end{aligned}
$$

\subsection{Optimization Model of CLRIP}

In CLRIP, the total costs consist of the location cost, inventory cost/and transportation cost. Location cost is the annual opening and operating costs of all opened DCs. Thus,

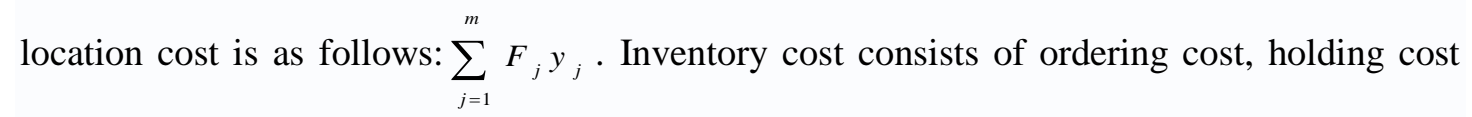
and backorder cost. Thus, it is given by: $\sum_{j=1}^{m}\left\{\frac{C_{3}}{T}+C_{h}\left(r_{j}-\tilde{\bar{D}}_{L, j}-\frac{1}{2} \tilde{\bar{D}}_{T, j}\right)+C\left(\bar{M}_{L+T, j}-T\right) y_{j}\right.$ [12]. Routing cost contains delivery cost from suppler to eachopened DC and distribution cost from each opened DC to customers. Therefore Q outing cost is as follows: $\sum_{j=1}^{m} \tilde{\bar{D}}_{j} C_{1} y_{j}+$ $\frac{1}{T} \sum_{g, h=1}^{n+m} \sum_{k=1}^{l} d_{g h} C_{x} x_{s h}$ According to the description and analysis above, the CLRIP model with FRD formulation as follows

$$
\begin{aligned}
& \min \tilde{\bar{C}}(r, T, x, y)=\sum_{j=1} F_{j} y_{j}+\sum_{j=1}^{m}\left\{\frac{C_{3}}{T}+C_{h}\left(r_{j}-\tilde{\bar{D}}_{L, j}-\frac{1}{2} \tilde{\bar{D}}_{T, j}\right)+\frac{C_{s}}{T} \bar{M}\left(\tilde{\bar{D}}_{L+T, j}-r_{j}\right)^{+}\right\} y_{j} \\
& \sqrt{-}+\sum_{j=1}^{m} \tilde{\overline{D_{j}}} C_{1} y_{j}+\frac{1}{T} \sum_{g, h=1}^{n+m} \sum_{k=1}^{l} d_{g h} C_{2} x_{g h k}
\end{aligned}
$$

$$
\begin{aligned}
& \sum_{g=1}^{n+m} \sum_{k=1}^{l} x_{g h k}=1, h=1,2, \cdots, n \\
& \sum_{g=1}^{n+m} \sum_{i=1}^{n} \tilde{\bar{d}}_{i} x_{g i k} \leq C_{q}, k=1,2, \cdots, l \\
& \sum_{g=1}^{m} \sum_{h=1}^{m} x_{g h k} \leq 1, k=1,2, \cdots, l
\end{aligned}
$$




$$
\begin{aligned}
& \sum_{g, h=1}^{n+m} x_{g h k}-\sum_{h, g=1}^{n+m} x_{h g k}=0, k=1,2, \cdots, l \\
& y_{j} \geq z_{i j}, i=1,2, \cdots, n, j=1,2, \cdots, m \\
& \tilde{\bar{D}}_{L+T, j}-\sum_{i=1}^{n} \widetilde{\widetilde{d}}_{L+T, i} z_{i j}=0, j=1,2, \cdots, m \\
& x_{g h k} \in\{0,1\}, g \neq h, g, h=1,2, \cdots, n+m, k=1,2, \cdots, l \\
& y_{j} \in\{0,1\}, j=1,2, \cdots, m \\
& z_{i j} \in\{0,1\}, i=1,2, \cdots, n, j=1,2, \cdots, m
\end{aligned}
$$

Formula (1) is the objective function to ensure that the total cost is the minimum. Constraint (2) guarantees that each customer has only one vehicle for its service. which is each customer in only one distribution route. Constraint (3) ensures that the customer's total demands of each distribution route cannot exceed the services capacity of the vehicle. Constraint (4) makes sure that each vehicle began at most one DC. Constraint (5) guarantees the continuity of distribution, if a vehicle enters a customer or DC node then it must leave from that node. Constraint (6) indicates that only the selected DC provides service for customers. Constraint (7) implies that the demand of each DC is the total demands of the customers allocated to it. Constraints (8), (9) and (10) are 0,1 degejsion variables.

\section{Deterministic Equivalent Form of CLRIP Mhodel with FRV}

As there are FRVs in CLRIP modế, it is more difficult to handle involving uncertainty problem. Thus, we need to conver this model to be deterministic. For the convenience of calculation, formula (1) is simplified before Conversion. Let $A$ be the certain costs that independent FRVs, let $\tilde{\overline{C_{1}}}$ be the backorder cost and $\tilde{\overline{C_{2}}}$ be the other costs associated with FRVs, thus

\subsection{Determination of Expected Shortage}

In practice, if customers' demands exceed the target inventory level in DC $j$ during the lead-time plus one period, then shortage occurs. In order to determine the expectation amount of shortage, we need to consider two cases, one is shown in Figure 2, and the other is shown in Figure 3 [9].

Case 1: When $\underline{D}_{L+T, j} \leq r_{j} \leq D_{L+T, j}$, the possibilistic mean values of the expected amount of shortage as follows 


$$
\bar{M}\left(\tilde{\bar{D}}_{L+T, j}-r_{j}\right)^{+}=\int_{0}^{1} \alpha\left(D_{L+T, j}^{+}\right)_{\alpha} d \alpha+\int_{L\left(r_{j}\right)}^{1} \alpha\left(D_{L+T, j}^{-}\right)_{\alpha} d \alpha-r_{j}\left(1-\frac{1}{2} L^{2}\left(r_{j}\right)\right)
$$

Case 2: When $D_{L+T, j} \leq r_{j} \leq \bar{D}_{L+T, j}$, the possibilistic mean values of the expected amount of shortage as follows:

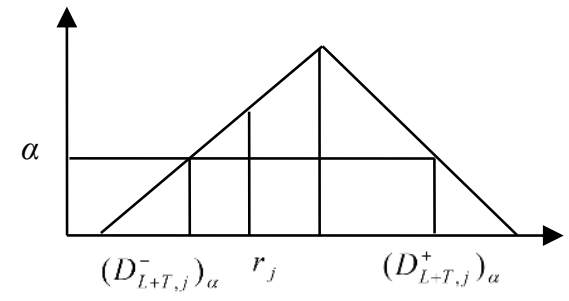

Figure 2. $\underline{D}_{L+T, j} \leq r_{j} \leq D_{L+T, j}$

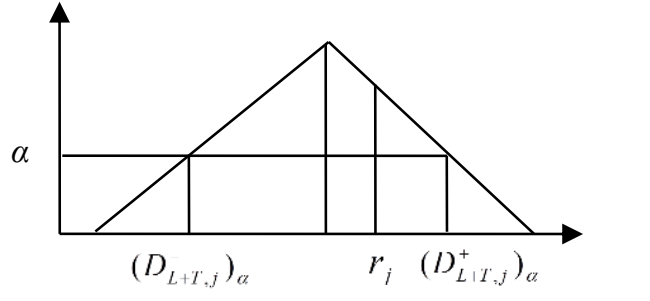

Figure 3. $D_{L+T, j} \leq r_{j} \leq D_{L+T, j}$

$$
\bar{M}\left(\tilde{\bar{D}}_{L+T, j}-r_{j}\right)^{+}=\int_{0}^{R\left(r_{j}\right)} \alpha\left(D_{L+T, j}^{+}\right)_{\alpha} d \alpha-\frac{1}{2} r_{j} R
$$

For more results on this topic, the work of $[9,12]$ may be referred to.

\subsection{Determination of the other Costs Expectations}

Due to $\tilde{\bar{C}}_{2}$ is FRV, its expectation is a unique fuzzy number. We will use possibilistic mean values of fuzzy number [12] to defuzzify Thus, FRV $\tilde{\bar{C}}_{2}$ can be transformed to deterministic one. The possibilistic mean values of $\overrightarrow{C_{2}}$ is given by

$$
\bar{M}\left(\tilde{\bar{C}} \geq \sum_{j=1}^{m}\left\{\left(C_{1}-Z C_{-}-\frac{T}{2} C_{h}\right) W_{j}\right\} y_{j}\right.
$$

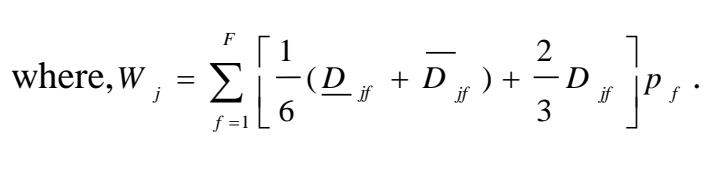

\subsection{Deterministic Equivalent Form of CLRIP Object Function}

Through the transforming above, the deterministic equivalent form of the object function in CLRIP model is as follows.

Case 1: When $\underline{D}_{L+T, j} \leq r_{j} \leq D_{L+T, j}$, deterministic equivalent object function is given by

$$
\begin{aligned}
\bar{M}(T, T, y) & =A+\frac{C_{s}}{T} \sum_{j=1}^{m}\left\{\int_{0}^{1} \alpha\left(D_{L+T, j}^{+}\right)_{\alpha} d \alpha+\int_{L\left(r_{j}\right)}^{1} \alpha\left(D_{L+T, j}^{-}\right)_{\alpha} d \alpha-r_{j}\left(1-\frac{1}{2} L^{2}\left(r_{j}\right)\right)\right\} y_{j} \\
& +\sum_{j=1}^{m}\left\{\left(C_{1}-L C_{h}-\frac{T}{2} C_{h}\right) W_{j}\right\}_{j} y_{j}
\end{aligned}
$$

Case 2: When $D_{L+T, j} \leq r_{j} \leq \bar{D}_{L+T, j}$, deterministic equivalent object function is given by

$$
\bar{M}(r, T, x, y)=A+\frac{C_{s}}{T} \sum_{j=1}^{m}\left\{\int_{0}^{R\left(r_{j}\right)} \alpha\left(D_{L+T, j}^{+}\right)_{\alpha} d \alpha-\frac{1}{2} r_{j} R^{2}\left(r_{j}\right)\right\} y_{j}
$$




$$
+\sum_{j=1}^{m}\left\{\left(C_{1}-L C_{h}-\frac{T}{2} C_{h}\right) W_{j}\right\} y_{j}
$$

\subsection{Deterministic Equivalent Forms of Constraints with FRVs}

Because there are FRVs in constraints (3) and (7), we need to transform them into deterministic. We can also use the possibilistic mean values of the FRD expectation to convert the FRVs in constraints above. Therefore, the deterministic equivalent form of constraint (3) can be given by

$$
\sum_{g=1}^{n+m} \sum_{i=1}^{n} \sum_{f=1}^{F} \frac{1}{6}\left(\underline{d}_{i f}+4 d_{i f}+\bar{d}_{i f}\right) p_{f} x_{g i k} \leq C_{q}, k=1,2, \cdots, l
$$

Likewise, the deterministic equivalent form of constraint (7) can be given by

$$
\begin{gathered}
\sum_{f=1}^{F} \frac{1}{6}\left(\underline{D}_{L+T, i f}+4 D_{L+T, i f}+\bar{D}_{L+T} \text { if }\right) p_{f} \\
-\sum_{i=1}^{n} \sum_{f=1}^{F} \frac{1}{6}\left(\underline{d}_{L+T, i f}+4 d_{L+T, i f}+\bar{d}_{L+T, i f} D_{f} z_{i j}=0, j=1,2, \cdots, m\right.
\end{gathered}
$$

\section{Solution Methodologies}

In this paper, the determined form of CLRIP model has four decision variables, such as $r_{j}$, $T$, $x$ and $y$. We need to determine the optimal values of $r_{j}$ and $T$, then to find the optimal values of $x$ and $y$.

\subsection{Determination of the Gptimal Value of $r_{j}$ and $T$}

Separately calculate the optimal values of $r_{j}$ and $T$ in two cases as 4.1 description.

Case 1: When $\underline{D}_{L+T, j} \leq r_{j} \leq D_{L+T, j}$, we have $\frac{\partial^{2} \bar{M}}{\partial r_{j}{ }^{2}}=\frac{C_{s}}{T}\left(\frac{r_{j}-\underline{D}_{L+T, j}}{\left(D_{L+T, j}-\underline{D}_{L+T, j}\right)^{2}}\right) \geq 0$, then

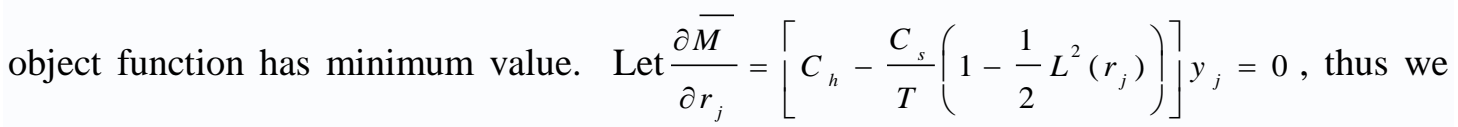
have $L^{2}\left(r_{j}\right)=2\left(1-\frac{T C_{h}}{C_{s}}\right)$.

Sinc $L\left(r_{j}\right)=\frac{r_{j}-\underline{D}_{L+T, j}}{D_{L+T, j}-\underline{D}_{L+T, j}}$, then we get $0 \leq L^{2}\left(r_{j}\right) \leq 1$, thus we have $\frac{C_{s}}{2 C_{h}} \leq T \leq \frac{C_{s}}{C_{h}}$.

And for $T$, we have $\frac{\partial \bar{M}}{\partial T}<0$, which means $\bar{M}(r, T, x, y)$ is strictly decreasing function of $T$, hence we get $T^{*}=\frac{C_{s}}{C_{h}}, r_{j}^{*}=\underline{D}_{L+T, j}$.

In this case, formula (17) can be converted to 


$$
\begin{aligned}
\bar{M}\left(r^{*}, T^{*}, x, y\right) & =A+\sum_{j=1}^{m}\left\{C_{h} \int_{0}^{1} \alpha\left(D_{L+T, j \alpha}^{+}+D_{L+T, j \alpha}^{-}\right) d \alpha\right\} y_{j} \\
& +\sum_{j=1}^{m}\left\{\left(C_{1}-L C_{h}-\frac{T}{2} C_{h}\right) W_{j}-C_{h} \underline{D}_{L+T, j}\right\} y_{j} \\
& =\sum_{j=1}^{m}\left[F_{j}+\frac{C_{h} C_{3}}{C_{s}}+\left(C_{1}+\frac{C_{s}}{2}\right) W_{j}\right]_{j}+\frac{C_{h}}{C_{s}} \sum_{g, h=1}^{n+m} \sum_{k=1}^{l} d_{g h} C_{2} x_{g h k}
\end{aligned}
$$

Case2: When $D_{L+T, j} \leq r_{j} \leq \bar{D}_{L+T, j}$, we have $\frac{\partial^{2} \bar{M}}{\partial r_{j}{ }^{2}}=\frac{C_{s}\left(\bar{D}_{L+T, j}-r_{j}\right)}{T\left(\bar{D}_{L+T, j}-D_{L+T, j}\right)^{2}} \geq 0$, then object function has minimum value, let $\frac{\partial \bar{M}}{\partial r_{j}}=\left[C_{h}-\frac{C_{s}}{2 T} R^{2}\left(r_{j}\right)\right] y_{j}=0$, thus we have $R^{2}\left(r_{j}\right)=\frac{2 T C_{h}}{C_{s}}$

Similarly, we have $0 \leq T \leq \frac{C_{s}}{2 C_{h}}, T^{*}=\frac{C_{s}}{2 C_{h}}, r^{*} D_{+T, j}$.

In this case, formula (18) can be converted to $\left.\bar{M}\left(r^{*}, T^{*}, x, y\right)=A+\sum_{j=1}^{m}\left(2 C_{h} \int_{0}^{1} \alpha D_{L}^{+} C_{j} d \alpha\right)_{j}-\sum_{h}^{m} D_{L+T, j}\right) y_{j}$ $+\sum_{j=1}^{m}\left\{\left(C_{1}-L Q_{h} 2 C_{s}^{s}\right) W_{j}\right\}_{3}$

$$
=\sum_{j=1}^{m}\left\{\left(-\frac{2 C_{h} C_{3}}{C_{s}}+\left(C_{t}-L C_{h}-\frac{C_{s}}{4}\right) W_{j}\right\}_{j} y_{j}+\frac{2 C_{h}}{C_{s}} \sum_{g, h=1}^{n+m} \sum_{k=1}^{l} d_{g h} C_{2} x_{g h k}\right.
$$

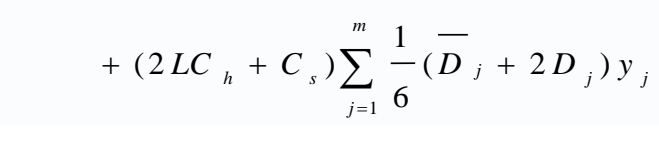

\subsection{Heuristic Method}

CLRIP has been proved a NP-hard problem [2], usually can be solved by heuristic method. Thus, this paper designed two-stage hybrid heuristic method to solve the CLRIP model with FRD [2, 4]. The heuristic algorithm can be divided into two stages. First stage is initial solution sage. Randomly assigned customers to each DC and vehicle, and then obtain the initial solution. Second stage is improvement stage. Based on Tabu Search (TS), firstly we wil improve the initial solution in location-allocation stage, and then we will continue to make improvements in the inventory-routing stage. The heuristic algorithm will stop when the termination criterion is matched. Finally, we will separately run the method in case 1 and case 2 , the smaller value of two cases will be the best solution.

5.2.1. Initial Solution Stage: Randomly generated initial solution in this stage.

Step1: Set $b=1, k=1, C_{q}=$ vehicle service capacity, Count $=0, V_{k}=\phi$. Put all customers into set $I$ and all DC into set $J$. 
Step2: Randomly select a customer $s$ from set $I$.

Step3: If demands of customer $s$ plus customers in $V_{k}$ less than or equal to $C_{q}$ ? If yes, go to Step 4. Otherwise, let $k=k+1$, then go to Step 4.

Step4: Put customer $s$ into $V_{k}$, delete customer $s$ from set $I$, if $I=\phi$ ? If yes, go to Step 5. Otherwise, go to Step 2.

Step5: Randomly select a DC from set $J$, put this DC into set $V_{b}$, delete this DC from set $J$, set $b=b+1$.

Step6: If $b>k$ ? If yes, go to Step 7. Otherwise, go to Step 5.

Step7: Compute the total cost $C$, set initial solution $C$ and $V_{b}$ as the current best solution, that is to say $x^{*}=x^{0}, C\left(x^{*}\right)=C\left(x^{0}\right)$.

5.2.2. Improvement Stage: Firstly, improve solution quality in distribution locationallocation stage.

Step8: Randomly generate $k$ routes in $k$ DCs which neither increase nor decrease new route than initial solution stage, and assigned the original customers to $k$ routs above.

Step9: If the total demands of each new route less than or equal to $C_{a}$ ? If yes, go to Step 10. Otherwise, go to Step 8.

Step10: If the move above in the tabu list? If yes, go to Step 11. Otherwise, go to Step 12.

Step11: If the length of tabu list equal to 7 [2]? If yes, go to Step 12. Otherwise, go to Step 8.

Step12: If $C\left(x^{1}\right) \leq C\left(x^{*}\right)$ ? If yes, (1) update $x^{*}=x^{l} \quad C\left(x^{*}\right)=C\left(x^{l}\right)$ (2) update $x^{0}=x^{l}, C\left(x^{0}\right)=C\left(x^{l}\right)$, (3)update the tabu list, (4) go to Step 13. Otherwise, (1) set no_improving_DC= no_improving_DC+1, (2) update $x_{0}=x^{1}, G\left(x^{0}\right)=C\left(x^{1}\right)$, (3) update the tabu list in location-allocation stage, (4) go to Step 13

Step13: If move times of no_improping_DC less than $M a x \_D C(=150)$ [2]? If yes, go to Step 8. Otherwise, go to Step 14.

Secondly, improve solution quality in inventory-routing stage. We use three candidate moves Move 1, Move 2 and Move 3in inventory-routing stage, Move 1 is insert operation, Move 2 is exchange operation and Move 3 is new-rout generation operation [2].

Move 1. Randomly select two routes $\mathrm{K}_{i}$ and $V_{j}$, then randomly select a customer $\mathrm{c}_{1}$ from $V_{i}$, find two customers who are the nearest ones to customer $\mathrm{c}_{1}$ from $V_{j}$, put customer $c_{1}$ into $V_{j}$, and distribution sequence is $c_{2}, c_{l}$ and $c_{3}$, then delete $c_{l}$ from $V_{i}$.

Move 2. Randomly select two routes $V_{i}$ and $V_{j}$, then randomly select a customer $c_{1}$ from $V_{i}$, find customer 2 who is the nearest one to customer $c_{1}$ from $V_{j}$, exchange $c_{1}$ and $c_{2}$.

Move 3. Randomly select one route $V_{i}$, then randomly select a customer $c_{l}$ from $V_{i}$, put $c_{l}$ into a new generation route $V_{j}$, then delete $c_{1}$ from $V_{i}$.

Step14: Randomly generate a move from the three moves above (from $x^{0}$ to $x^{l}$ ).

Step15: If the demands of route $V_{i}$ and route $V_{j}$ separately less than or equal to $C_{q}$ ? If yes, go to Step 16. Otherwise, go to Step 14.

Step16-If the length of tabu list equal to 7 [2]? If yes, go to Step 17. Otherwise, go to Step 14.

Step 17: If $C\left(x^{1}\right) \leq C\left(x^{*}\right)$ ? If yes, (1) update $x^{*}=x^{l}, C\left(x^{*}\right)=C\left(x^{l}\right)$, (2) update $x^{0}=x^{1}, C\left(x^{0}\right)=C\left(x^{l}\right)$, (3)update the tabu list, (4) go to Step 18. Otherwise, (1) set no_improving_Routing= no_improving_Routing +1 , (2) update $x^{0}=x^{I}, C\left(x^{0}\right)=C\left(x^{l}\right)$, (3) update the tabu list in inventory-routing stage, (4) go to Step 18.

Step18: If move times of no_improving_Routing less than Max_Routing (=2000) [2]? If yes, go to Step 14. Otherwise, go to Step 19.

Step19: If the termination criterion is matched i.e. Count=max_Count $(=5)$ [2]? If yes, stop. 
Otherwise, go to Step 8.

\section{Computational Experiments}

In order to verify the effectiveness of our heuristic algorithm, we assume that there are 6 DCs and 12 customers in distribution system. The following parameters are determined, the unit commodity transportation cost from supplier to each DC is 2 , the unit distance transportation cost from every DC to each customer is 3, the cost per order placed to the supplier by each DC is 15 , the lead time of each DC is 10 days, vehicle service capacity is 50 , inventory holding cost per unit commodity per year is 0.5 , backorder cost per unit commodity per time is 10 . As 5.1 mentioned, order time $T$ is $C_{s}$ divided by $C_{h}$ which equal to 20 days in case 1 and 10 days in case 2 .

As mentioned above, we have

$C_{l}=2, C_{2}=3, C_{3}=15, L=10$ days $=1 / 36$ year $, C_{q}=50, C_{h}=0.5, C_{s}=10$

Case 1: $T=C_{s} / C_{h}=20$ days=1/18year, Case2: $T=C_{s} / 2 C_{h}=10$ days=1/36year

Parameters of DCs and customers are separately shown in Tables 1 and Table 2.

Table 1. Parameters of DCs

\begin{tabular}{c|c|c|c|c|c|c}
\hline $\mathrm{DC} j$ & 1 & 2 & 3 & 4 & 5 & 6 \\
\hline Coordinate & $(15,19)$ & $(19,75)$ & $(31,87)$ & $(71,41)$ & $(61,83)$ & $(59,51)$ \\
\hline $\mathrm{F}_{j}$ & 320 & 290 & 460 & 200 & 430 & 450 \\
\hline
\end{tabular}

Table 2. Parameters of CUstomers

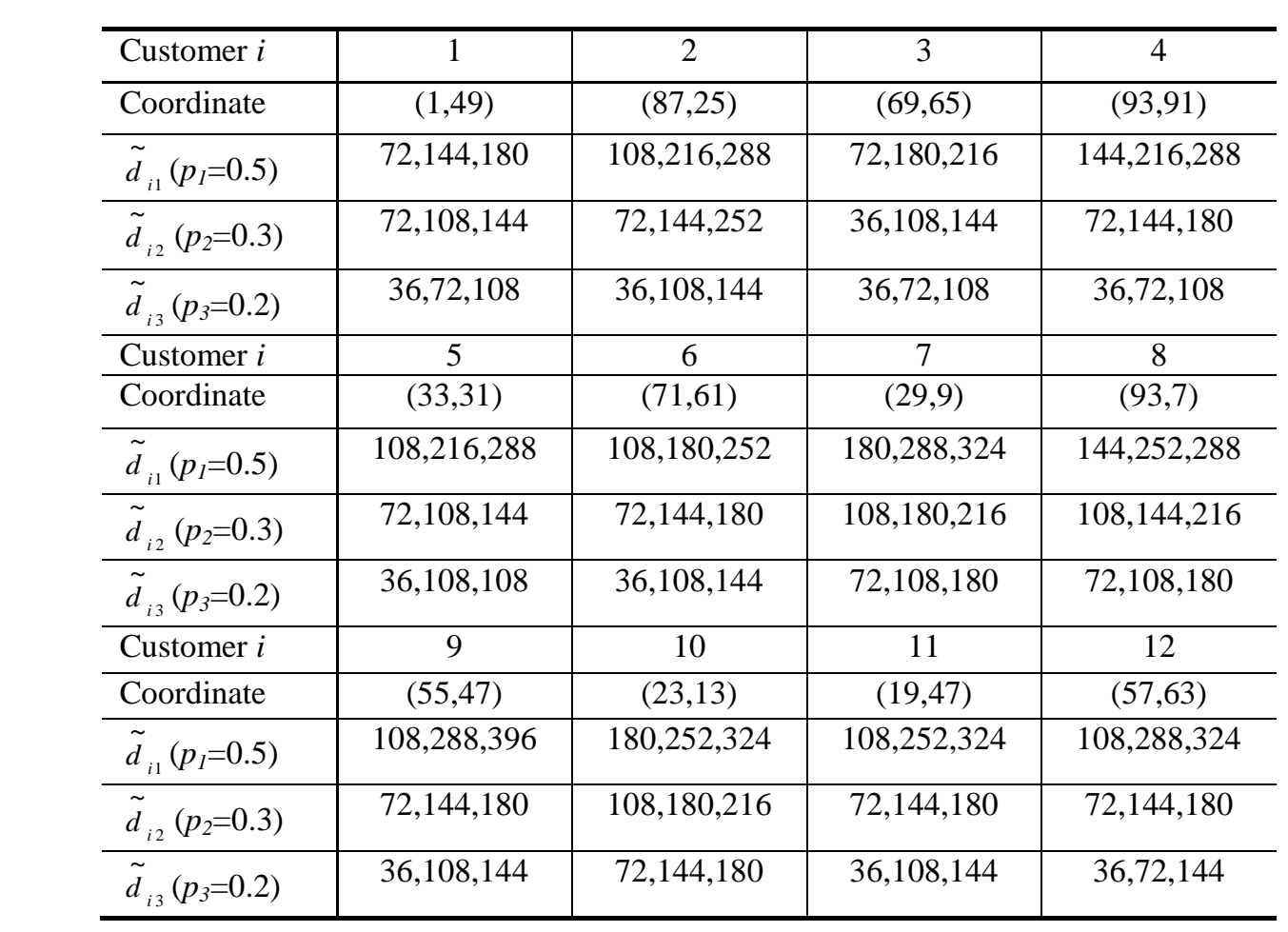

By using the method in 5.2, Matlog toolbox of Matlab7.0 is used to develop the algorithm procedure. Since TS is a random search algorithm, then the quality of its best solution may be related to the initial solution. Therefore, we separately solve and record the 10 groups of best solutions when the initial solution is different [18], in order to test our model and algorithm of 
CLRIP. Due to that routes of the opened DCs and vehicles are different, initial solution is different, then the best total cost and the corresponding opened DCs and delivery routes are as shown in Table 3. Where, $J_{j}$ denotes DC $j, I_{i}$ denotes customer $i$, for example, distribution route $J_{5}=\left\{I_{6}, I_{2}, I_{4}\right\}$ denotes that distribution sequence is $J_{5}, I_{6}, I_{2}, I_{4}$ and $J_{5}$.

From Table 3, when different initial solutions are given, each best total cost is much closed via using our heuristic algorithm, and the relative deviation between maximum and minimum is only $0.45 \%$, which verified the effectiveness of the proposed two-stage heuristic algorithm.

Table 3. Initial Solution and Best Solution

\begin{tabular}{|c|c|c|c|c|c|}
\hline \multicolumn{3}{|c|}{ Initial solution } & \multicolumn{3}{|c|}{ Best solution } \\
\hline $\begin{array}{c}\text { Opened } \\
\text { DC }\end{array}$ & Distribution routs & $\begin{array}{l}\text { Total } \\
\text { cost }\end{array}$ & $\begin{array}{l}\text { Opened } \\
\text { DC }\end{array}$ & Distribution routs & Total \\
\hline$J_{2}, J_{3}, J_{4}, J_{6}$ & $\begin{array}{l}J_{2}=\left\{I_{1}, I_{2}, I_{3}, I_{4}\right\}, J_{3}=\left\{I_{8},\right. \\
\left.I_{9}, I_{10}\right\} J_{4}=\left\{I_{5}, I_{6}, I_{7}\right\}, J_{6}= \\
\left\{I_{11}, I_{12}\right\}\end{array}$ & 6967.0 & $\begin{array}{l}J_{1}, J_{3}, J_{4} \\
J_{5}, J_{6}\end{array}$ & $\begin{aligned} J_{1} & =\left\{I_{1}, I_{10}, I_{5}\right\}, J_{3}=\left\{I_{9}, I_{11}, I_{7}\right\}, \\
J_{4} & =\left\{I_{12}\right\}, J_{5}=\left\{I_{6}, I_{4}\right\}, \\
J_{6} & =\left\{I_{8}, I_{2}, I_{3}\right\}\end{aligned}$ & 6732.1 \\
\hline$J_{1}, J_{2}, J_{3}, J_{5}$ & $\begin{array}{l}J_{1}=\left\{I_{1}, I_{2}, I_{3}, I_{4}\right\}, J_{2}=\left\{I_{5},\right. \\
\left.I_{6}, I_{7}\right\}, J_{3}=\left\{I_{11}, I_{12}\right\}, \\
J_{5}=\left\{I_{8}, I_{9}, I_{10}\right\}\end{array}$ & 7086.2 & $\begin{array}{l}J_{1}, J_{2}, J \\
J_{4} \\
J_{6}\end{array}$ & $\begin{array}{l}J_{I}=\left\{I_{70}, I_{\}}, J_{2}=\left\{I_{1}, I_{11},\right.\right. \\
J_{3}=\left\{I_{2,} I_{12}, I_{5}\right\}, J_{4}=\left\{I_{9},\right.\end{array}$ & 6734.9 \\
\hline$J_{1}, J_{3}, J_{4}, J_{6}$ & $\begin{array}{l}J_{1}=\left\{I_{11}, I_{12}\right\}, J_{3}=\left\{I_{8}, I_{9},\right. \\
\left.I_{10}\right\} J_{4}=\left\{I_{5}, I_{6}, I_{7}\right\}, \\
J_{6}=\left\{I_{1}, I_{2}, I_{3}, I_{4}\right\}\end{array}$ & 7008.9 & & $\begin{aligned} J_{1} & =\left\{I_{5}, I_{7}, I_{9}\right\}, J_{3}=\left\{I_{1}, I_{4}, I_{3}\right\}, \\
J_{4} & =\left\{I_{12}, I_{6}\right\}, S_{5}=\left\{I_{11}\right\}, \\
J_{6} & =\left\{I_{2}, I_{9}\right\}\end{aligned}$ & 6726.1 \\
\hline$J_{2}, J_{3}, J_{5}, J_{6}$ & $\begin{array}{l}J_{2}=\left\{I_{1}, I_{2}, I_{3}, I_{4}\right\}, J_{3}=\left\{I_{5},\right. \\
\left.I_{6}, I_{7}\right\}, J_{5}=\left\{I_{8}, I_{9}, I_{10}\right\}, \\
J_{6}=\left\{I_{11}, I_{12}\right\}\end{array}$ & & & $\begin{array}{l}J_{l}=\left\{I_{16} I_{7}\right\}, J_{2}=\left\{I_{5}, I_{12}, I_{4}\right\}, \\
J_{3}=\left\{I_{1}, I_{11}, I_{2}\right\}, J_{4}=\left\{I_{9}, I_{3}, I_{8}\right\}, \\
J_{6}=\left\{I_{6}\right\}\end{array}$ & 6743.5 \\
\hline $\begin{array}{l}J_{l}, J_{2}, J_{3}, \\
J_{5}\end{array}$ & $\begin{array}{l}J_{I}=\left\{I_{1}, I_{2}, I_{3}, I_{4}\right\}, J_{2}=\{l \\
\left.I_{9}, I_{10}\right\}, J_{3}=\left\{I_{5}, I_{6}, I_{7}\right\}, \\
J_{5}=\left\{I_{11}, I_{12}\right\}\end{array}$ & & $\begin{array}{l}J_{1}, J_{2}, \sqrt{3} \\
\left(4, J_{6}\right.\end{array}$ & $\begin{aligned} J_{I} & =\left\{I_{10}, I_{7}\right\}, J_{2}=\left\{I_{6}, I_{12}\right\} \\
J_{3} & =\left\{I_{1}, I_{11}, I_{5},\right\}, J_{4}=\left\{I_{2}, I_{4}\right\}, \\
J_{6} & =\left\{I_{8}, I_{3}, I_{9}\right\}\end{aligned}$ & 6725.1 \\
\hline $\begin{array}{l}J_{1}, J_{2}, J_{4}, \\
J_{5}\end{array}$ & $\begin{array}{l}J_{1}=\left\{I_{1}, I_{2}, I_{3}, I_{4}\right\} J_{3}=\left\{I_{5},\right. \\
\left.I_{6}, I_{7}\right\}, J_{4}=\left\{I_{14}, I_{2}\right\} \\
J_{5}=\left\{I_{8}, I_{9}, I_{0}\right\}\end{array}$ & & $\begin{array}{l}J_{1}, J_{2}, J_{3} \\
J_{4}, J_{5}, J_{6}\end{array}$ & $\begin{array}{l}J_{1}=\left\{I_{10}\right\}, J_{2}=\left\{I_{11}, I_{7}, I_{4}\right\}, \\
J_{3}=\left\{I_{1}\right\}, J_{4}=\left\{I_{2}, I_{8}, I_{5}\right\} \\
J_{5}=\left\{I_{6}, I_{3}\right\}, J_{6}=\left\{I_{9}, I_{12}\right\}\end{array}$ & 6751.2 \\
\hline & & 7031.7 & $\begin{array}{l}J_{1}, J_{2}, J_{4}, \\
J_{5}, J_{6}\end{array}$ & $\begin{array}{l}J_{1}=\left\{I_{5}, I_{7}\right\}, J_{2}=\left\{I_{1}, I_{11}, I_{3}, I_{9}\right\}, \\
J_{4}=\left\{I_{2}\right\}, J_{3}=\left\{I_{6}, I_{8}, I_{4}\right\}, \\
J_{6}=\left\{I_{12}, I_{10}\right\}\end{array}$ & 6732.8 \\
\hline$J_{1}, J_{2}, J_{3}, J_{5}$ & $\begin{array}{l}I_{7}=\left\{I_{8}, I_{9}, I_{10}\right\}, J_{2}=I_{5}, \\
\left.I_{6}, I_{7}\right\}, J_{3}=\left\{J_{1}, I_{2}\right\}, \\
J_{5}=\left\{I_{f}, I_{2},\left(3, I_{4}\right\}\right.\end{array}$ & 7053.0 & $\begin{array}{l}J_{1}, J_{2}, J_{4}, \\
J_{5}, J_{6}\end{array}$ & $\begin{array}{l}J_{1}=\left\{I_{10}, I_{7}, I_{5}\right\}, J_{2}=\left\{I_{6}\right\}, \\
J_{4}=\left\{I_{9}, I_{2}\right\}, J_{5}=\left\{I_{12}, I_{8}, I_{4}\right\} \\
J_{6}=\left\{I_{1}, I_{11}, I_{3}\right\}\end{array}$ & 6731.5 \\
\hline $\begin{array}{l}J_{l}, J_{2}, J_{4}, \\
J_{6}\end{array}$ & $\begin{array}{l}J_{I}=\left\{I_{1} I_{6}, I_{7}\right\}, J_{2}=\left\{I_{8}, I_{9},\right. \\
\left.I_{10}\right\} J_{4}=\left\{I_{1}, I_{2}, I_{3}, I_{4}\right\}, \\
I_{6}=\left\{I_{11}, I_{12}\right\}\end{array}$ & 6812.4 & $\begin{array}{l}J_{1}, J_{3}, J_{4}, \\
J_{5}, J_{6}\end{array}$ & $\begin{array}{l}J_{1}=\left\{I_{10}, I_{1}, I_{7}\right\}, J_{3}=\left\{I_{3}\right\}, \\
J_{4}=\left\{I_{2}, I_{8}, I_{9}\right\}, J_{5}=\left\{I_{11}, I_{4}, I_{6}\right\}, \\
J_{6}=\left\{I_{5}, I_{12}\right\}\end{array}$ & 6734.5 \\
\hline & $\begin{array}{l}J_{I}=\left\{I_{5}, I_{6}, I_{7}\right\}, J_{3}=\left\{I_{8}, I_{9},\right. \\
\left.I_{10}\right\} J_{4}=\left\{I_{1}, I_{2}, I_{3}, I_{4}\right\}, \\
J_{5}=\left\{I_{11}, I_{12}\right\}\end{array}$ & 6973.3 & $\begin{array}{l}J_{2}, J_{3}, J_{4}, \\
J_{5}, J_{6}\end{array}$ & $\begin{array}{l}J_{2}=\left\{I_{1}, I_{5}, I_{11}\right\} \\
J_{3}=\left\{I_{9}, I_{3}\right\}, J_{4}=\left\{I_{10}, I_{7}, I_{12}\right\} \\
J_{5}=\left\{I_{4}, I_{8}, I_{2}\right\}, J_{6}=\left\{I_{6}\right\}\end{array}$ & 6755.6 \\
\hline
\end{tabular}

Therefore, the proposed model and algorithm can efficiently reduce the total cost of distribution system, so as to realize the integrated optimization of $\mathrm{B} 2 \mathrm{C}$ E-Commerce distribution system.

\section{Conclusions}

In $\mathrm{B} 2 \mathrm{C}$ E-Commerce distribution environment, considering customer demands FRV, this paper makes a study on the integrated decisions of three levels with location, vehicle routing 
and inventory control. The mixed 0-1 integer programming model for CLRIP with FRVs is established to minimize the total system cost, the expected value and the possibilistic mean value are used to convert this model into deterministic. A two-stage hybrid heuristic algorithm based on TS is proposed to solve this problem. The experiment shows that the model is effective, and the algorithm is reliable to solve above-mentioned problems.

The distribution network studied in this paper assumes that each DC has one and only one vehicle and capacity of each DC is the same. But in fact, each DC may have more than one vehicle and various vehicle types, even the different capacity of each DC. Thus, in further research, the model can be extended to multi-vehicle, a variety of vehicle types and each DC with different capacity.

\section{Acknowledgements}

This work is partially supported by the Humanities and Social Sciences Projeet of the Education Department of Heilongjiang Province of China. (No.12522084), the Science and Technology Research Projects of the Education Department of Heilongjiang Province of China (No.12531138) and the National Natural Science Foundation of China (No.11271103).

\section{References}

[1] S. C. Liu and S. B. Lee, "A two-phase heuristic method for the multi-depot location routing problem taking inventory control decisions into consideration", International Joumal of Advanced Manufacturing Technology, vol.22, no.11-12, (2003), pp. 941-950.

[2] S. C. Liu and C. C. Lin, "A heuristic method for the combined location routing and inventory problem", International Journal of Advanced Manufacturing Technology, 16126, no.4, (2005), pp. 372-381.

[3] M. Z. J. Shen and L. Qi, "Incorporating inventory and routng costs in strategic location models", European Journal of Operational Research, vol.179, no.2, (2007), pp.372-389.

[4] A. A. Javid and N. Azad, "Incorporating location, outing and inventory decisions in supply chain network design", Transportation Research Pan E: Logistics and Transportation Review, vol.46, no.5, (2010), pp.582597.

[5] F. Fatemeh and T. M. Reza Using a genetic algorithm to optimize the total cost for a location-routinginventory problem in a Supply chain with risk pooling", Journal of Applied Operational Research, vol.4, no.1, (2012), pp.2-13.

[6] C. F. Wang and B. Shuai, "Location-inventory-routing problem of maintenance spare parts logistics system with lateral transs hipment", Computer Engineering and Applications, vol.49, no.14, (2013), pp.10-14.

[7] G. B. Cui and Y. J. Li, "Cornbined location routing and inventory problem with fuzzy demand in logistics system", Control and Decision, vol.22, no.9, (2007), pp.1000-1004.

[8] H. Kwakernaak, "Fuzzy random variables-I: Definition and theorems", Information Sciences, vol.15, (1978), pp.1-29.

[9] Oshmita and D Chakraborty, "Fuzzy periodic review system with fuzzy random variable demand", European Journal of Operational Research, vol.198, no.1, (2009), pp.113-120.

[10] S. Bag, D. Chakraborty and A. R. Roy, "A production inventory model with fuzzy random demand and with flexibility and reliability considerations", Computers \& Industrial Engineering, vol.56, no.1, (2009), pp.411416.

[11] L. Li, Order quantity model for VMI under fuzzy random environment of supply and demand", Statistics and Decision, vol.27, no.7, (2011), pp.58-61.

[12] Oshmita and D. Chakraborty, "A fuzzy random periodic review system with variable lead-time and negative crashing cost", Applied Mathematical Modeling, vol.36, no.12, (2012), pp.6312-6322.

[13] D. Panda, M. Rong and M. Maiti, "Fuzzy mixture two warehouse inventory model involving fuzzy random variable lead time demand and fuzzy total demand", Central European Journal of Operations Research, vol.22, no.1, (2014), pp.187-209.

[14] J. P. Xu and F. Yan and S. Li, "Vehicle routing optimization with soft time windows in a fuzzy random environment", Transportation Research Part E, vol.47, no.6, (2011), pp.1075-1091.

[15] J. P. Xu and J. Gang, "Multi-objective bi-level construction material transportation scheduling in large-scale construction projects under a fuzzy random environment", Transportation Planning and Technology, vol.36, no.4, (2013), pp.352-376. 
[16] Y. K. Liu and B. D. Liu, "Fuzzy random variables: A scalar expected value operator", Fuzzy Optimization and Decision Making, vol.2, no.2, (2003), pp.143-160.

[17] B. D. Liu, R. Q. Zhao and G. Wang, "Uncertain Programming with applications", Tsinghua University Press, Peking, (2003).

[18] J. Qin, L. L. Ni and L. X. Miao, "Optimization model and combined simulated annealing algorithm for multi-level multi-commodity logistics network design”, Application Research of Computers, vol.27, no.9, (2010), pp.3348-3351.

\section{Authors}

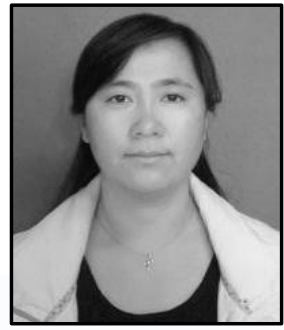

Dehui Chen is a Ph.D. candidate in management science and engineering at Harbin University of Science and Technology. She is currently a lecture of School of Computer and Information Engineering at Harbin University of Commerce in China. Her main research interests are E-Commerce logistics system analysis and optimization, Supply Chain Management.

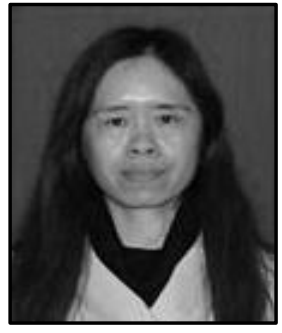

Dongyan Chen received Ph.D. degrea from Harbin Institute of Technology, major in Aircraft design in 2000. She is currently a professor and doctor tutor of School of Applied Science at Harbin University of Science and Technology in China. Her main research interests are system analysis and optimization, robust control of time delay systems.
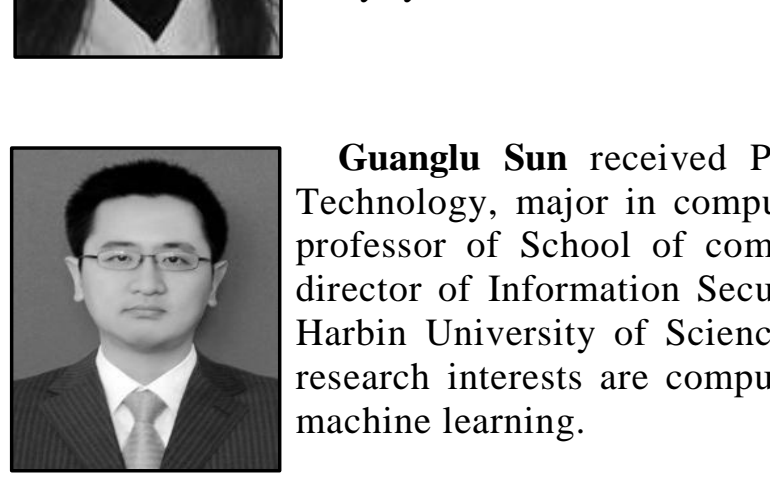

Guanglu Sun received Ph.D. degree from Harbin Institute of Techology, major in computer science in 2008 . He is currently a professor of School of computer science and technology and the dinector of Information Security and Intelligent Technology Lab at Harbin University of Science and Technology in China. His main research interests are computer networks, information security and machinelearning.

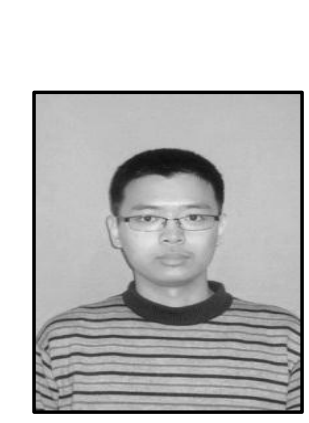

Guangming Liu is a postgraduate student in computer science and technology at Harbin University of Science and Technology in China. His main research interests are machine learning, and feature selection. 